\title{
Research Article \\ Existence of Positive Solutions of Fourth-Order Problems with Integral Boundary Conditions
}

\author{
Ruyun Ma and Tianlan Chen \\ Department of Mathematics, Northwest Normal University, Lanzhou 730070, China \\ Correspondence should be addressed to Ruyun Ma, ruyun_ma@126.com \\ Received 5 May 2010; Accepted 7 July 2010 \\ Academic Editor: Daniel Franco
}

Copyright @ 2011 R. Ma and T. Chen. This is an open access article distributed under the Creative Commons Attribution License, which permits unrestricted use, distribution, and reproduction in any medium, provided the original work is properly cited.

We study the existence of positive solutions of the following fourth-order boundary value problem with integral boundary conditions, $u^{(4)}(t)=f\left(t, u(t), u^{\prime \prime}(t)\right), t \in(0,1), u(0)=\int_{0}^{1} g(s) u(s) d s, u(1)=$ $0, u^{\prime \prime}(0)=\int_{0}^{1} h(s) u^{\prime \prime}(s) d s, u^{\prime \prime}(1)=0$, where $f:[0,1] \times[0,+\infty) \times(-\infty, 0] \rightarrow[0,+\infty)$ is continuous, $g, h \in L^{1}[0,1]$ are nonnegative. The proof of our main result is based upon the Krein-Rutman theorem and the global bifurcation techniques.

\section{Introduction}

The deformations of an elastic beam in an equilibrium state, whose both ends are simple supported, can be described by the fourth-order boundary value problem

$$
\begin{gathered}
u^{(4)}(t)=f\left(t, u(t), u^{\prime \prime}(t)\right), \quad t \in(0,1), \\
u(0)=u(1)=u^{\prime \prime}(0)=u^{\prime \prime}(1)=0,
\end{gathered}
$$

where $f:[0,1] \times \mathbb{R} \times \mathbb{R} \rightarrow \mathbb{R}$ is continuous; see Gupta [1,2]. In the past twenty more years, the existence of solutions and positive solutions of these kinds of problems and the Lidstone problem has been extensively studied; see [3-9] and the references therein. In [3], Ma was concerned with the existence of positive solutions of (1.1) and (1.2) under the assumptions:

(H1) $f:[0,1] \times[0,+\infty) \times(-\infty, 0] \rightarrow[0,+\infty)$ is continuous and there exist constants $a, b, c, d \in[0,+\infty)$, with $a+b>0, c+d>0$ such that

$$
f(t, u, p)=a u-b p+o(|(u, p)|), \quad \text { as }|(u, p)| \longrightarrow 0
$$


uniformly for $t \in[0,1]$, and

$$
f(t, u, p)=c u-d p+o(|u, p|), \quad \text { as }|(u, p)| \longrightarrow \infty
$$

uniformly for $t \in[0,1]$, where $|(u, p)|:=\sqrt{u^{2}+p^{2}}$;

(H2) $f(t, u, p)>0$ for $t \in[0,1]$ and $(u, p) \in([0,+\infty) \times(-\infty, 0]) \backslash\{(0,0)\}$;

(H3) there exist constants $a_{0}, b_{0} \in[0,+\infty)$ satisfying $a_{0}^{2}+b_{0}^{2}>0$ and

$$
f(t, u, p) \geq a_{0} u-b_{0} p, \quad(t, u, p) \in[0,1] \times[0,+\infty) \times(-\infty, 0] .
$$

Ma proved the following.

Theorem A (see [3, Theorem 4.1]). Let (H1), (H2), and (H3) hold. Assume that either

$$
\mu_{1}(c, d)<1<\mu_{1}(a, b)
$$

or

$$
\mu_{1}(a, b)<1<\mu_{1}(c, d),
$$

where $\mu_{1}(\alpha, \beta)$ denotes the first generalized eigenvalue of the generalized eigenvalue problem

$$
\begin{gathered}
u^{(4)}(t)=\mu\left(\alpha u(t)-\beta u^{\prime \prime}(t)\right), \quad t \in(0,1), \\
u(0)=u(1)=u^{\prime \prime}(0)=u^{\prime \prime}(1)=0 .
\end{gathered}
$$

Then (1.1) and (1.2) have at least one positive solution.

At the same time, we notice that a class of boundary value problems with integral boundary conditions appeared in heat conduction, chemical engineering underground water flow, thermoelasticity, and plasma physics. Such a kind of problems include two-point, threepoint, multipoint and nonlocal boundary value problems as special cases and attracting the attention of a few readers; see [10-13] and the references therein. For example, In particular, Zhang and Ge [10] used Guo-Krasnoselskii fixed-point theorem to study existence and nonexistence of positive solutions of the following fourth-order boundary value problem with integral boundary conditions:

$$
\begin{gathered}
u^{(4)}(t)=w(t) f\left(t, u(t), u^{\prime \prime}(t)\right), \quad t \in(0,1), \\
u(0)=\int_{0}^{1} g(s) u(s) d s, \quad u(1)=0, \\
u^{\prime \prime}(0)=\int_{0}^{1} h(s) u^{\prime \prime}(s) d s, \quad u^{\prime \prime}(1)=0,
\end{gathered}
$$


where $w$ may be singular at $t=0$ and (or) $t=1 ; f:[0,1] \times[0,+\infty) \times(-\infty, 0] \rightarrow[0,+\infty)$ is continuous, and $g, h \in L^{1}[0,1]$ are nonnegative.

Motivated by $[3,10]$, in this paper, we consider the existence of positive solutions of the following fourth-order boundary value problem with integral boundary conditions:

$$
\begin{array}{rlrl}
u^{(4)}(t) & =f\left(t, u(t), u^{\prime \prime}(t)\right), & t \in(0,1), \\
u(0) & =\int_{0}^{1} g(s) u(s) d s, & & u(1)=0, \\
u^{\prime \prime}(0) & =\int_{0}^{1} h(s) u^{\prime \prime}(s) d s, & & u^{\prime \prime}(1)=0,
\end{array}
$$

under the assumption

(H4) $g, h \in L^{1}[0,1]$ are nonnegative, and $\|g\|_{1}:=\int_{0}^{1} g(s) d s \leq \pi / 4, v:=\int_{0}^{1}(1-s) h(s) d s<1$. The main result of this paper is the following.

Theorem 1.1. Let (H1), (H2), (H3), and (H4) hold. Assume that either

$$
\lambda_{1}(c, d)<1<\lambda_{1}(a, b)
$$

or

$$
\lambda_{1}(a, b)<1<\lambda_{1}(c, d)
$$

where

$$
\begin{gathered}
u^{(4)}(t)=\lambda\left(\alpha u(t)-\beta u^{\prime \prime}(t)\right), \quad t \in(0,1), \\
u(0)=\int_{0}^{1} g(s) u(s) d s, \quad u(1)=0, \\
u^{\prime \prime}(0)=\int_{0}^{1} h(s) u^{\prime \prime}(s) d s, \quad u^{\prime \prime}(1)=0 .
\end{gathered}
$$

Then (1.9) has at least one positive solution.

Remark 1.2. Theorem 1.1 generalizes [3, Theorem 4.1] where the special case $g=0$ and $h=0$ was treated.

Remark 1.3. Zhang and Ge [10] proved existence and nonexistence of positive solutions via Guo-Krasnoselskii fixed-point theorem under some conditions which do not involve the eigenvalues of (1.12)-(1.14). While our Theorem 1.1 is established under (1.10) or (1.11) which is related to the eigenvalues of (1.12)-(1.14). Moreover, (1.10) and (1.11) are optimal. Let us consider the problem

$$
\begin{gathered}
u^{(4)}(x)=\pi^{4} u(x)+1, \quad x \in(0,1), \\
u(0)=u(1)=u^{\prime \prime}(0)=u^{\prime \prime}(1)=0 .
\end{gathered}
$$


In this case, $\lambda_{1}\left(\pi^{4}, 0\right)=1$ and the corresponding eigenfunction is $\varphi_{1}=\sin \pi x$. However, (1.15) and (1.16) has no positive solution. (In fact, suppose on the contrary that (1.15) and (1.16) has a positive solution $u$. Multiplying (1.15) with $\sin \pi x$ and integrating from 0 to 1 , we get a desired contradiction!).

Suppose that $E$ is a real Banach space with norm $\|\cdot\|$. Let $K$ be a cone in $E$. A nonlinear mapping $A:[0, \infty) \times K \rightarrow E$ is said to be positive if $A([0, \infty) \times K) \subseteq K$. It is said to be $K$ completely continuous if $A$ is continuous and maps bounded subsets of $[0, \infty) \times K$ to precompact subset of $E$. Finally, a positive linear operator $V$ on $E$ is said to be a linear minorant for $A$ if $A(\lambda, u) \geq \lambda V(x)$ for $(\lambda, u) \in[0, \infty) \times K$. If $B$ is a continuous linear operator on $E$, denote $r(B)$ the spectral radius of $B$. Define

$$
c_{K}(B)=\{\lambda \in[0, \infty): \text { there exists } x \in K \text { with }\|x\|=1, x=\lambda B x\} .
$$

The following lemma will play a very important role in the proof of our main results, which is essentially a consequence of Dancer [14, Theorem 2].

Lemma 1.4. Assume that

(i) $K$ has nonempty interior and $E=\overline{K-K}$;

(ii) $A:[0, \infty) \times K \rightarrow E$ is $K$-completely continuous and positive, $A(\lambda, 0)=0$ for $\lambda \in \mathbb{R}$, $A(0, u)=0$ for $u \in K$ and

$$
A(\lambda, u)=\lambda B u+F(\lambda, u),
$$

where $B: E \rightarrow E$ is a strongly positive linear compact operator on $E$ with the spectral radius $r(B)>0$, $F:[0, \infty) \times K \rightarrow$ E satisfies $\|F(\lambda, u)\|=\circ(\|u\|)$ as $\|u\| \rightarrow 0$ locally uniformly in $\lambda$.

Then there exists an unbounded connected subset $\mathcal{C}$ of

$$
\Phi_{K}(A)=\{(\lambda, u) \in[0, \infty) \times K: u=A(\lambda, u), u \neq 0\} \cup\left\{\left(r(B)^{-1}, 0\right)\right\}
$$

such that $\left(r(B)^{-1}, 0\right) \in \mathcal{C}$.

Moreover, if $A$ has a linear minorant $V$ and there exists a

$$
(\mu, y) \in(0, \infty) \times K
$$

such that $\|y\|=1$ and $\mu V y \geq y$, then $C$ can be chosen in

$$
\Phi_{K}(A) \cap([0, \mu] \times K)
$$

Proof. Since $B$ is a strongly positive compact endomorphism of $E$ and $P$ has nonempty interior, we have from Amann [15, Theorem 3.2] that the set $c_{K}(B)$ in [14, Theorem 2] reduces to a single point $\left(r(B)^{-1}, 0\right)$. Now the desired result is a consequence of Dancer [14, Theorem 2]. 
The rest of the paper is arranged as follows. In Section 2, we state and prove some preliminary results about the spectrum of (1.12)-(1.14). Finally, in Section 3, we proved our main result.

\section{Generalized Eigenvalues}

Lemma 2.1 (see [10]). Assume that (H4) holds. Then for any $y \in C[0,1]$, the boundary value problem

$$
\begin{gathered}
-u^{\prime \prime}(t)=y(t), \quad t \in(0,1), \\
u(0)=\int_{0}^{1} g(s) u(s) d s, \quad u(1)=0
\end{gathered}
$$

has a unique solution $u$ which is given by

$$
u(t)=\int_{0}^{1} H_{1}(t, s) y(s) d s, \quad t \in[0,1]
$$

where

$$
\begin{gathered}
H_{1}(t, s)=G(t, s)+\frac{1-t}{1-\mu} \int_{0}^{1} G(s, \tau) g(\tau) d \tau, \quad \mu:=\int_{0}^{1}(1-s) g(s) d s, \\
G(t, s)= \begin{cases}s(1-t), & 0 \leq s \leq t \leq 1, \\
t(1-s), & 0 \leq t \leq s \leq 1 .\end{cases}
\end{gathered}
$$

Lemma 2.2 (see [10]). Assume that (H4) holds. Then for any $y \in C[0,1]$, the boundary value problem

$$
\begin{gathered}
u^{(4)}(t)=y(t), \quad t \in(0,1), \\
u(0)=\int_{0}^{1} g(s) u(s) d s, \quad u(1)=0, \\
u^{\prime \prime}(0)=\int_{0}^{1} h(s) u^{\prime \prime}(s) d s, \quad u^{\prime \prime}(1)=0
\end{gathered}
$$

has a unique solution $u$ which is given by

$$
u(t)=\iint_{0}^{1} H_{1}(t, x) H_{2}(x, s) y(s) d s d x, \quad t \in[0,1]
$$


where

$$
H_{2}(x, s)=G(x, s)+\frac{1-x}{1-v} \int_{0}^{1} G(s, \tau) h(\tau) d \tau .
$$

Lemma 2.3 (see [10]). Assume that (H4) holds. Then one has

$$
\begin{aligned}
& H_{1}(t, s)>0, \quad H_{2}(t, s)>0, \quad G(t, s)>0, \quad t, s \in(0,1), \\
& H_{1}(t, s) \geq 0, \quad H_{2}(t, s) \geq 0, \quad G(t, s) \geq 0, \quad t, s \in[0,1] .
\end{aligned}
$$

Let

(H5) $(\alpha, \beta) \in[0,+\infty) \times[0,+\infty)$ be two given constants with $\alpha+\beta>0$.

Definition 2.4. One says that $\lambda$ is a generalized eigenvalue of linear problem

$$
\begin{gathered}
u^{(4)}(t)=\lambda\left(\alpha u(t)-\beta u^{\prime \prime}(t)\right), \quad t \in(0,1), \\
u(0)=\int_{0}^{1} g(s) u(s) d s, \quad u(1)=0, \\
u^{\prime \prime}(0)=\int_{0}^{1} h(s) u^{\prime \prime}(s) d s, \quad u^{\prime \prime}(1)=0,
\end{gathered}
$$

if (2.8) and (2.9) have nontrivial solutions.

Let

$$
e(t):=\cos \frac{\pi}{2} t, \quad t \in[0,1]
$$

Let $Y=C[0,1]$ with the norm $\|u\|_{\infty}=\max _{t \in[0,1]}|u(t)|$. Let $Z=L^{1}[0,1]$ with the norm $\|u\|_{1}=\int_{0}^{1}|u(s)| d s$.

Let

$$
X=\left\{u \in C^{2}[0,1] \mid u \text { satisfies }(2.9), \exists \varepsilon>0, \text { s. t. }-\varepsilon e(t) \leq-u^{\prime \prime}(t) \leq \varepsilon e(t), t \in[0,1]\right\}
$$

For $u \in X$, from Lemma 2.1, it follows that

$$
u(t)=\int_{0}^{1} H_{1}(t, s)\left(-u^{\prime \prime}(s)\right) d s, \quad t \in[0,1]
$$


By simple calculations, we have

$$
\begin{aligned}
\int_{0}^{1} H_{1}(t, s) e(s) d s & =\int_{0}^{1} G(t, s) e(s) d s+\frac{1-t}{1-\mu} \int_{0}^{1}\left[\int_{0}^{1} G(s, \tau) g(\tau) d \tau\right] e(s) d s \\
& =\frac{4}{\pi^{2}} e(t)-\frac{4(1-t)}{\pi^{2}}+\frac{1-t}{1-\mu} \int_{0}^{1}\left[\int_{0}^{1} G(s, \tau) g(\tau) d \tau\right] e(s) d s \\
& \leq \frac{4}{\pi^{2}} e(t)-\frac{4(1-t)}{\pi^{2}}+\frac{1-t}{1-\mu} \int_{0}^{1} G(s, s) e(s) d s \int_{0}^{1} g(\tau) d \tau \\
& =\frac{4}{\pi^{2}} e(t)-\frac{4(1-t)}{\pi^{2}}+\frac{4(1-t)}{\pi^{2}} \frac{\|g\|_{1}}{1-\mu}\left(\frac{4}{\pi}-1\right) .
\end{aligned}
$$

Combining this with $(\mathrm{H} 4)$, we conclude that

$$
\int_{0}^{1} H_{1}(t, s) e(s) d s \leq \frac{4}{\pi^{2}} e(t), \quad t \in[0,1] .
$$

This together with $(2.12)$ and the fact that $-\varepsilon e(t) \leq-u^{\prime \prime}(t) \leq \varepsilon e(t)$ imply that

$$
-\frac{4}{\pi^{2}} \varepsilon e(t) \leq u(t) \leq \frac{4}{\pi^{2}} \varepsilon e(t), \quad t \in[0,1] .
$$

Since $\left(4 / \pi^{2}\right) \varepsilon<\varepsilon$, we may define the norm of $u \in X$ by

$$
\|u\|_{X}:=\inf \left\{\varepsilon \mid-\varepsilon e(t) \leq-u^{\prime \prime}(t) \leq \varepsilon e(t), t \in[0,1]\right\} .
$$

We claim that $\left(X,\|\cdot\|_{X}\right)$ is a Banach space.

In fact, let $\left\{y_{n}\right\} \subset X$ be a Cauchy sequence, that is, $\left\|y_{n}-y_{m}\right\|_{X} \rightarrow 0$ as $n, m \rightarrow \infty$. From the definition of $\|\cdot\|_{X}$, it follows that

$$
\left\|y_{n}-y_{m}\right\|_{C^{2}} \leq c^{*}\left\|y_{n}-y_{m}\right\|_{X^{\prime}} \text { for some constant } c^{*}>0,
$$

where $\|\cdot\|_{C^{2}}$ is a normal in $X$ defined by $\|y\|_{C^{2}}:=\max \left\{\left|-y^{\prime \prime}(t)\right| \mid t \in[0,1]\right\}$. Thus,

$$
\left\|y_{n}-y_{m}\right\|_{C^{2}} \longrightarrow 0, \quad \text { as } m, n \longrightarrow \infty
$$

By the completeness of $\left(C^{2}[0,1],\|\cdot\|_{C^{2}}\right)$, there exists $y^{*} \in C^{2}[0,1]$, such that

$$
\left\|y_{n}-y^{*}\right\|_{C^{2}} \longrightarrow 0, \quad \text { as } n \longrightarrow \infty \text {. }
$$

From the fact that $\left\|y_{n}-y_{m}\right\|_{X} \rightarrow 0$, we have that for arbitrary $\epsilon>0$, there exists $N>0$, such that

$$
\left\|y_{n}-y_{m}\right\|_{X} \leq \epsilon, \quad \text { whenever } m, n>N
$$


and subsequently,

$$
-\epsilon e(t) \leq y_{n}(t)-y_{m}(t) \leq \epsilon e(t), \quad \text { whenever } m, n>N
$$

Fixed $n$ and let $m \rightarrow \infty$, we get

$$
-\epsilon e(t) \leq y_{n}(t)-y^{*}(t) \leq \epsilon e(t), \quad \text { whenever } n>N .
$$

This is,

$$
\left\|y_{n}-y^{*}\right\|_{X} \leq \epsilon, \quad \text { whenever } n>N
$$

Therefore, $\left(X,\|\cdot\|_{X}\right)$ is a Banach space.

Let

$$
P:=\left\{u \in X \mid u(t) \geq 0, u^{\prime \prime}(t) \leq 0, t \in[0,1]\right\} .
$$

Then the cone $P$ is normal and nonempty interior $P^{0}$ and $X=\overline{P-P}$.

In fact, for any $u \in X$, it follows from the definition of $X$ that

(1) there exist real number $a>0$, such that

$$
\left|u^{\prime \prime}(t)\right| \leq a e(t), \quad t \in[0,1]
$$

(2) $u(1)=0, u(0)=\int_{0}^{1} g(s) u(s) d s$.

From $u(0)=\int_{0}^{1} g(s) u(s) d s$ and $(\mathrm{H} 4)$, we obtain that $u^{\prime}\left(t^{*}\right)=0$ for some $t^{*} \in(0,1)$. Moreover,

$$
\left|u^{\prime}(t)\right|=\left|u^{\prime}\left(t^{*}\right)+\int_{t^{*}}^{t} u^{\prime \prime}(s) d s\right| \leq a
$$

and subsequently,

$$
|u(t)|=\left|u(1)-\int_{t}^{1} u^{\prime}(s) d s\right| \leq|u(1)|+\int_{t}^{1}\left|u^{\prime}(s)\right| d s \leq a(1-t) \leq \hat{a} e(t), \quad t \in[0,1],
$$

for some $\widehat{a}>0$. We may take $\widehat{a}$ satisfying

$$
\widehat{a}>\frac{4 a}{\pi^{2}}
$$


Boundary Value Problems

Now, let us define

$$
\begin{gathered}
y(t)=\hat{a} e(t), \\
z(t)=u(t)+y(t) .
\end{gathered}
$$

Then $y, z \in P$, and

$$
u=z(t)-y(t)
$$

Thus, $X \subseteq \overline{P-P}$. Obviously, $\overline{P-P} \subseteq X$.

Lemma 2.5. Assume that (H4) holds. Then for any $u \in X$, one has

$$
\|u\|_{\infty}<\left\|u^{\prime \prime}\right\|_{\infty} \leq\|u\|_{X}
$$

Proof. In fact, for $u \in X$, we have that

$$
\begin{aligned}
u(t) & =\int_{0}^{1} H_{1}(t, s)\left(-u^{\prime \prime}(s)\right) d s \\
& \leq\left\|u^{\prime \prime}\right\|_{\infty} \int_{0}^{1} H_{1}(t, s) d s \\
& =\left\|u^{\prime \prime}\right\|_{\infty} \int_{0}^{1}\left[G(t, s)+\frac{1-t}{1-\mu} \int_{0}^{1} G(s, \tau) g(\tau) d \tau\right] d s \\
& \leq\left\|u^{\prime \prime}\right\|_{\infty}\left[\int_{0}^{1} G(s, s) d s+\frac{1}{1-\mu} \int_{0}^{1} G(s, s) d s \int_{0}^{1} g(\tau) d \tau\right] \\
& =\left\|u^{\prime \prime}\right\|_{\infty}\left[\frac{1}{6}+\frac{1}{6} \frac{\|g\|_{1}}{1-\mu}\right]
\end{aligned}
$$

From $\mu \leq\|g\|_{1} \leq \pi / 4$, we have that $1 / 6+(1 / 6)\left(\|g\|_{1} /(1-\mu)\right)<1$, and so $|u(t)|<\left\|u^{\prime \prime}\right\|_{\infty}$, and accordingly $\|u\|_{\infty}<\left\|u^{\prime \prime}\right\|_{\infty}$.

We have from the fact that $-\varepsilon e(t) \leq-u^{\prime \prime}(t) \leq \varepsilon e(t), t \in[0,1]$, that

$$
\left|u^{\prime \prime}(t)\right| \leq \varepsilon e(t) \leq \varepsilon,
$$

which implies that $\left\|u^{\prime \prime}\right\|_{\infty} \leq \varepsilon$, and consequently $\left\|u^{\prime \prime}\right\|_{\infty} \leq\|u\|_{X}$.

For $u \in X$, define a linear operator $T: X \rightarrow Y$ by

$$
T u(t)=\iint_{0}^{1} H_{1}(t, x) H_{2}(x, s)\left[\alpha u(s)-\beta u^{\prime \prime}(s)\right] d s d x, \quad t \in[0,1]
$$


Theorem 2.6. Assume that (H4) and (H5) hold. Let $r(T)$ be the spectral radius of $T$. Then (2.8) and (2.9) has an algebraically simple eigenvalue, $\lambda_{1}(\alpha, \beta)=(r(T))^{-1}$, with a positive eigenfunction $\varphi_{1}(\cdot) \in P^{0}$. Moreover, there is no other eigenvalue with a positive eigenfunction.

Remark 2.7. If $g=h \equiv 0$, then $\lambda_{1}(\alpha, \beta)$ can be explicitly given by

$$
\lambda_{1}(\alpha, \beta)=\frac{\pi^{4}}{\alpha+\beta \pi^{2}}
$$

and the corresponding eigenfunction $\varphi_{1}(t)=\sin \pi t, t \in[0,1]$.

Proof of Theorem 2.6. From Lemma 2.2, it is easy to check that (2.8) and (2.9) is equivalent to the integral equation

$$
u(t)=\lambda T u(t), \quad t \in[0,1]
$$

We claim that $T: X \rightarrow X$.

In fact, for $u \in X$, we have that

$$
\begin{aligned}
\left|-(T u)^{\prime \prime}(t)\right| & =\left|\int_{0}^{1} H_{2}(t, s)\left[\alpha u(s)-\beta u^{\prime \prime}(s)\right] d s\right| \\
& \leq\left(\alpha\|u\|_{\infty}+\beta\left\|u^{\prime \prime}\right\|_{\infty}\right) \int_{0}^{1} H_{2}(t, s) d s \\
& :=C_{0} \int_{0}^{1} H_{2}(t, s) d s .
\end{aligned}
$$

Since

$$
\begin{aligned}
\int_{0}^{1} H_{2}(t, s) d s & =\int_{0}^{1} G(t, s) d s+\frac{1-t}{1-v} \iint_{0}^{1} G(s, \tau) h(\tau) d \tau d s \\
& =\frac{t(1-t)}{2}+(1-t) \frac{\iint_{0}^{1} G(s, \tau) h(\tau) d \tau d s}{1-v} \\
& \leq(1-t)\left[\frac{1}{2}+\frac{\iint_{0}^{1} G(s, \tau) h(\tau) d \tau d s}{1-v}\right] \\
& :=\Gamma_{0}(1-t),
\end{aligned}
$$

and for some constant $\rho_{1}>0$, it concludes that

$$
\rho_{1} e(t) \leq 1-t \leq e(t), \quad t \in[0,1]
$$


Hence,

$$
-\sigma e(t) \leq-(T u)^{\prime \prime}(t) \leq \sigma e(t), \quad t \in[0,1]
$$

where $\sigma:=\Gamma_{0} C_{0}$, it follows that $T u \in X$, and accordingly $T(X) \subseteq X$.

If $u \in P$, then $\alpha u(t)-\beta u^{\prime \prime}(t) \geq 0$ on $t \in[0,1]$, and accordingly

$$
\begin{gathered}
(T u)^{\prime \prime}(t)=-\int_{0}^{1} H_{2}(t, s)\left[\alpha u(s)-\beta u^{\prime \prime}(s)\right] d s \leq 0, \quad t \in[0,1], \\
(T u)(t)=\int_{0}^{1} H_{1}(t, s)\left(-(T u)^{\prime \prime}(s)\right) d s \geq 0, \quad t \in[0,1] .
\end{gathered}
$$

Thus $T u \in P$, and accordingly $T(P) \subseteq P$.

Now, since $T(X) \subseteq C^{4}[0,1] \cap X$, and $C^{4}[0,1] \cap X$ is compactly embedded in $X$, we have that $T: X \rightarrow X$ is compact.

Next, we show that $T: P \rightarrow P$ is positive.

For $u \in P \backslash\{0\}$, if $\alpha>0$, from Lemma 2.3, we have

$$
\begin{aligned}
-(T u)^{\prime \prime}(t) & \geq \alpha \int_{0}^{1} H_{2}(t, s) u(s) d s \\
& =\alpha\left[\int_{0}^{1} G(t, s) d s+\frac{1-t}{1-v} \int_{0}^{1}\left(\int_{0}^{1} G(s, \tau) h(\tau) d \tau\right) u(s) d s\right] \\
& \geq \frac{\alpha \int_{0}^{1}\left[\int_{0}^{1} G(s, \tau) h(\tau) d \tau\right] u(s) d s}{1-v}(1-t) \\
& :=\Gamma_{3}(1-t) .
\end{aligned}
$$

Combining this with (2.39), there exist $r_{2}:=\Gamma_{3} \rho_{1}>0$ such that

$$
-(T u)^{\prime \prime}(t) \geq r_{2} e(t), \quad t \in[0,1]
$$

For $u \in P \backslash\{0\}$, if $\beta>0$, applying a similar proof process of (2.43), we have

$$
\begin{aligned}
-(T u)^{\prime \prime}(t) & \geq \beta \int_{0}^{1} H_{2}(t, s)\left[-u^{\prime \prime}(s)\right] d s \\
& \geq \frac{\beta \int_{0}^{1}\left[\int_{0}^{1} G(s, \tau) h(\tau) d \tau\right]\left(-u^{\prime \prime}(s)\right) d s}{1-\mathcal{v}}(1-t) \\
& :=\Gamma_{4}(1-t) .
\end{aligned}
$$


Combining this with (2.39), there exist $r_{3}:=\Gamma_{4} \rho_{1}>0$ such that

$$
-(T u)^{\prime \prime}(t) \geq r_{3} e(t), \quad t \in[0,1] .
$$

This together with (2.9) and (H4) imply $u(t) \geq 0$ on $[0,1]$.

Therefore, it follows from (2.43) and (2.45) that $T u \in P^{0}$.

Now, by the Krein-Rutman theorem ([16, Theorem 7.C]; [17, Theorem 19.3]), $T$ has an algebraically simple eigenvalue $r(T)>0$ with an eigenfunction $\varphi_{1}(\cdot) \in P^{0}$. Moreover, there is no other eigenvalue with a positive eigenfunction. Correspondingly, $\lambda_{1}(\alpha, \beta)=(r(T))^{-1}$ with a positive eigenfunction of $\varphi_{1}(\cdot)$, is a simple eigenvalue of (2.8) and (2.9). Moreover, for (2.8) and (2.9), there is no other eigenvalue with a positive eigenfunction.

\section{The Proof of the Main Result}

Before proving Theorem 1.1, we denote $L: D(L) \rightarrow Y$ by setting

$$
L u:=u^{\prime \prime \prime \prime}, \quad u \in D(L),
$$

where

$$
D(L)=\left\{u \in C^{4}[0,1] \mid u(0)=\int_{0}^{1} g(s) u(s) d s, u^{\prime \prime}(0)=\int_{0}^{1} h(s) u^{\prime \prime}(s) d s, u(1)=u^{\prime \prime}(1)=0\right\} .
$$

It is easy to check that $L^{-1}: Y \rightarrow X$ is compact.

Let $\zeta, \xi \in C([0,1] \times[0,+\infty) \times(-\infty, 0])$ be such that

$$
\begin{aligned}
& f(t, u, p)=a u-b p+\zeta(t, u, p) \\
& f(t, u, p)=c u-d p+\xi(t, u, p) .
\end{aligned}
$$

Obviously,(H1) implies that

$$
\begin{aligned}
& \lim _{|(u, p)| \rightarrow 0} \frac{\zeta(t, u, p)}{|(u, p)|}=0 \quad \text { uniformly for } t \in[0,1] \\
& \lim _{|(u, p)| \rightarrow \infty} \frac{\xi(t, u, p)}{|(u, p)|}=0 \quad \text { uniformly for } t \in[0,1]
\end{aligned}
$$

Let

$$
\tilde{\xi}(r)=\max \{|\xi(t, u, p)||0 \leq|(u, p) \mid \leq r, t \in[0,1]\},
$$


then $\tilde{\xi}$ is nondecreasing and

$$
\lim _{r \rightarrow \infty} \frac{\tilde{\xi}(r)}{r}=0
$$

Let us consider

$$
L u=\lambda\left(a u-b u^{\prime \prime}\right)+\lambda \zeta\left(t, u, u^{\prime \prime}\right), \quad \lambda>0
$$

as a bifurcation problem from the trivial solution $u \equiv 0$. It is to easy to check that (3.8) can be converted to the equivalent equation

$$
\begin{aligned}
u(t)= & \lambda\left\{\iint_{0}^{1} H_{1}(t, x) H_{2}(x, s)\left[a u(s)-b u^{\prime \prime}(s)\right] d s d x\right\} \\
& +\lambda\left\{\iint_{0}^{1} H_{1}(t, x) H_{2}(x, s) \zeta\left(s, u(s), u^{\prime \prime}(s)\right) d s d x\right\} \\
:= & A(\lambda, u)(t) .
\end{aligned}
$$

From the proof process of Theorem 2.6, the operator $B: X \rightarrow X$,

$$
B u(t):=\iint_{0}^{1} H_{1}(t, x) H_{2}(x, s)\left[a u(s)-b u^{\prime \prime}(s)\right] d s d x
$$

is compact and strongly positive. Define $F:[0,+\infty) \times X \rightarrow X$ by

$$
F(\lambda, u):=\lambda \iint_{0}^{1} H_{1}(t, x) H_{2}(x, s) \zeta\left(s, u(s), u^{\prime \prime}(s)\right) d s d x
$$

then we have from (3.4) and Lemma 2.5 that

$$
\|F(\lambda, u)\|_{X}=o\left(\|u\|_{X}\right), \quad \text { as }\|u\|_{X} \longrightarrow 0,
$$

locally uniformly in $\lambda$. From(H2) and Theorem 2.6 (with obvious changes), it follows that if $(\lambda, u)$ is a nontrivial solution of (3.8) with $\lambda>0$, then $u \in P^{0}$. Combining this with Lemma 1.4, we conclude that there exists an unbounded connected subset $C$ of the set

$$
\left\{(\lambda, u) \in(0, \infty) \times P \mid u=A(\lambda, u), u \in P^{0}\right\} \cup\left\{\left(\lambda_{1}(a, b), 0\right)\right\}
$$

such that $\left(\lambda_{1}(a, b), 0\right) \in \mathcal{C}$. 
Proof of Theorem 1.1. It is clear that any solution of the form $(1, u)$ yields a solution $u$ of $(1.9)$. We will show that $\mathcal{C}$ crosses the hyperplane $\{1\} \times X$. To do this, it is enough to show that $\mathcal{C}$ joins $\left(\lambda_{1}(a, b), 0\right)$ to $\left(\lambda_{1}(c, d), \infty\right)$. Let $\left(\mu_{n}, y_{n}\right) \in \mathcal{C}$ satisfy

$$
\mu_{n}+\left\|y_{n}\right\|_{X} \longrightarrow \infty, \quad n \longrightarrow \infty,
$$

we note that $\mu_{n}>0$ for all $n \in \mathbb{N}$ since $(0,0)$ is the only solution of (3.8) for $\lambda=0$ and $\mathcal{C} \cap(\{0\} \times X)=\emptyset$.

Case $1\left(\lambda_{1}(c, d)<1<\lambda_{1}(a, b)\right)$. In this case, we show that

$$
\left(\lambda_{1}(c, d), \lambda_{1}(a, b)\right) \subseteq\{\lambda \in \mathbb{R} \mid \exists(\lambda, u) \in \mathcal{C}\} .
$$

We divide the proof into two steps.

Step 1. We show that if there exists a constant number $M>0$ such that

$$
\mu_{n} \in(0, M]
$$

then $\mathcal{C}$ joins $\left(\lambda_{1}(a, b), 0\right)$ to $\left(\lambda_{1}(c, d), \infty\right)$.

From (3.16), we have that $\left\|y_{n}\right\|_{X} \rightarrow \infty$. We divide the equation

$$
L y_{n}=\mu_{n}\left(c y_{n}-d y_{n}^{\prime \prime}\right)+\mu_{n} \xi\left(t, y_{n}, y_{n}^{\prime \prime}\right)
$$

by $\left\|y_{n}\right\|_{X}$ and set $\overline{y_{n}}=y_{n} /\left\|y_{n}\right\|_{X}$. Since $\overline{y_{n}}$ is bounded in $X$, choosing a subsequence and relabeling if necessary, we see that $\overline{y_{n}} \rightarrow \bar{y}$ for some $\bar{y} \in X$ with $\|\bar{y}\|_{X}=1$. Moreover, we have from (3.7) and Lemma 2.5 that

$$
\lim _{n \rightarrow \infty} \frac{\left|\xi\left(t, y_{n}(t), y_{n}^{\prime \prime}(t)\right)\right|}{\left\|y_{n}\right\|_{X}}=0
$$

Since $\left|\xi\left(t, y_{n}(t), y_{n}^{\prime \prime}(t)\right)\right| /\left\|y_{n}\right\|_{X} \leq \tilde{\xi}\left(\sqrt{2}\left\|y_{n}\right\|_{X}\right) /\left\|y_{n}\right\|_{X}$. Thus,

$$
\bar{y}(t)=\iint_{0}^{1} H_{1}(t, x) H_{2}(x, s) \bar{\mu}\left[c \bar{y}(s)-d \bar{y}^{\prime \prime}(s)\right] d s d x,
$$

where $\bar{\mu}:=\lim _{n \rightarrow \infty} \mu_{n}$, again choosing a subsequence and relabeling if necessary. Thus,

$$
L \bar{y}(t)=\bar{\mu}\left(c \bar{y}(t)-d \bar{y}^{\prime \prime}(t)\right), \quad t \in[0,1] .
$$

This together with Theorem 2.6 imply that $\bar{\mu}=\lambda_{1}(c, d)$. Therefore, $\mathcal{C}$ joins $\left(\lambda_{1}(a, b), 0\right)$ to $\left(\lambda_{1}(c, d), \infty\right)$.

Step 2. We show that there exists a constant $M$ be such that $\mu_{n} \in(0, M]$ for all $n$. 
By Lemma 1.4, we only need to show that $A$ has a linear minorant $V$ and there exists a $(\mu, y) \in(0, \infty) \times P$ such that $\|y\|_{X}=1$ and $\mu V y \geq y$.

$\operatorname{By}(\mathrm{H} 3)$, there exist constants $a_{0}, b_{0} \in[0,+\infty)$ satisfying $a_{0}^{2}+b_{0}^{2}>0$ and

$$
f(t, u, p) \geq a_{0} u-b_{0} p,(t, u, p) \in[0,1] \times[0,+\infty) \times(-\infty, 0] .
$$

For $u \in X$, let

$$
V u(t)=\iint_{0}^{1} H_{1}(t, x) H_{2}(x, s)\left[a_{0} u(s)-b_{0} u^{\prime \prime}(s)\right] d s d x
$$

then $V$ is a linear minorant of $A$. Moreover,

$$
\begin{aligned}
V\left(\frac{4}{\pi^{2}} e(t)\right) & =\iint_{0}^{1} H_{1}(t, x) H_{2}(x, s)\left[a_{0} \frac{4}{\pi^{2}} e(s)+b_{0} e(s)\right] d s d x \\
& =\left(\frac{4}{\pi^{2}} a_{0}+b_{0}\right) \iint_{0}^{1} H_{1}(t, x) H_{2}(x, s) e(s) d s d x \\
& =\left(\frac{4}{\pi^{2}} a_{0}+b_{0}\right) \iint_{0}^{1}\left[G(t, x)+\frac{1-t}{1-\mu} \int_{0}^{1} G(x, \tau) g(\tau) d \tau\right] H_{2}(x, s) e(s) d s d x \\
& \geq\left(\frac{4}{\pi^{2}} a_{0}+b_{0}\right) \frac{\iint_{0}^{1}\left[\int_{0}^{1} G(x, \tau) g(\tau) d \tau\right] H_{2}(x, s) e(s) d s d x}{1-\mu}(1-t) \\
& :=\Gamma_{5} \frac{4}{\pi^{2}}(1-t)
\end{aligned}
$$

where

$$
\Gamma_{5}=\frac{\left(a_{0}+\left(\pi^{2} / 4\right) b_{0}\right) \iint_{0}^{1}\left[\int_{0}^{1} G(x, \tau) g(\tau) d \tau\right] H_{2}(x, s) e(s) d s d x}{1-\mu}
$$

Combining this with (2.39), we conclude that $\lambda V\left(\left(4 / \pi^{2}\right) e(t)\right) \geq\left(4 / \pi^{2}\right) e(t)$, here, $\lambda:=$ $\left(\Gamma_{5} \rho_{1}\right)^{-1}$. Therefore, we have that from Lemma 1.4 that $\left|\mu_{n}\right| \leq \lambda$.

Case $2\left(\lambda_{1}(a, b)<1<\lambda_{1}(c, d)\right)$. In this case, if $\left(\mu_{n}, y_{n}\right) \in \mathcal{C}$ such that

$$
\lim _{n \rightarrow \infty}\left(\mu_{n}+\left\|y_{n}\right\|_{X}\right)=\infty
$$

and $\lim _{n \rightarrow \infty} \mu_{n}=\infty$, then

$$
\left(\lambda_{1}(a, b), \lambda_{1}(c, d)\right) \subseteq\{\lambda \in(0, \infty) \mid(\lambda, u) \in \mathcal{C}\}
$$

and, moreover, $(\{1\} \times X) \cap \mathcal{C} \neq \emptyset$. 
Assume that there exists $M>0$, such that for all $n \in \mathbb{N}$,

$$
\mu_{n} \in(0, M]
$$

Applying a similar argument to that used in Step 1 of Case 1, after taking a subsequence and relabeling if necessary, it follows that

$$
\left(\mu_{n}, y_{n}\right) \longrightarrow\left(\lambda_{1}(c, d), \infty\right), \quad n \longrightarrow \infty
$$

Again $\mathcal{C}$ joins $\left(\lambda_{1}(a, b), 0\right)$ to $\left(\lambda_{1}(c, d), \infty\right)$ and the result follows.

\section{Acknowledgments}

The authors are very grateful to the anonymous referees for their valuable suggestions. This paper was supported by the NSFC (no. 11061030), the Fundamental Research Funds for the Gansu Universities.

\section{References}

[1] C. P. Gupta, "Existence and uniqueness theorems for the bending of an elastic beam equation," Applicable Analysis, vol. 26, no. 4, pp. 289-304, 1988.

[2] C. P. Gupta, "Existence and uniqueness results for the bending of an elastic beam equation at resonance," Journal of Mathematical Analysis and Applications, vol. 135, no. 1, pp. 208-225, 1988.

[3] R. Ma, "Existence of positive solutions of a fourth-order boundary value problem," Applied Mathematics and Computation, vol. 168, no. 2, pp. 1219-1231, 2005.

[4] R. Ma and L. Xu, "Existence of positive solutions of a nonlinear fourth-order boundary value problem," Applied Mathematics Letters, vol. 23, no. 5, pp. 537-543, 2010.

[5] Y. Li, "Positive solutions of fourth-order boundary value problems with two parameters," Journal of Mathematical Analysis and Applications, vol. 281, no. 2, pp. 477-484, 2003.

[6] R. Ma and H. Wang, "On the existence of positive solutions of fourth-order ordinary differential equations," Applicable Analysis, vol. 59, no. 1-4, pp. 225-231, 1995.

[7] Z. Bai and H. Wang, "On positive solutions of some nonlinear fourth-order beam equations," Journal of Mathematical Analysis and Applications, vol. 270, no. 2, pp. 357-368, 2002.

[8] R. Ma and J. Xu, "Bifurcation from interval and positive solutions of a nonlinear fourth-order boundary value problem," Nonlinear Analysis: Theory, Methods E Applications, vol. 72, no. 1, pp. 113122, 2010.

[9] Y. Ma, "Existence of positive solutions of Lidstone boundary value problems," Journal of Mathematical Analysis and Applications, vol. 314, no. 1, pp. 97-108, 2006.

[10] X. Zhang and W. Ge, "Positive solutions for a class of boundary-value problems with integral boundary conditions," Computers E Mathematics with Applications, vol. 58, no. 2, pp. 203-215, 2009.

[11] J. M. Gallardo, "Second-order differential operators with integral boundary conditions and generation of analytic semigroups," The Rocky Mountain Journal of Mathematics, vol. 30, no. 4, pp. 1265-1291, 2000.

[12] G. L. Karakostas and P. Ch. Tsamatos, "Multiple positive solutions of some integral equations arisen from nonlocal boundary-value problems," Electronic Journal of Differential Equations, vol. 30, pp. 1-17, 2002.

[13] A. Lomtatidze and L. Malaguti, "On a nonlocal boundary value problem for second order nonlinear singular differential equations," Georgian Mathematical Journal, vol. 7, no. 1, pp. 133-154, 2000.

[14] E. N. Dancer, "Global solution branches for positive mappings," Archive for Rational Mechanics and Analysis, vol. 52, pp. 181-192, 1973.

[15] H. Amann, "Fixed point equations and nonlinear eigenvalue problems in ordered Banach spaces," SIAM Review, vol. 18, no. 4, pp. 620-709, 1976. 
[16] E. Zeidler, Nonlinear Functional Analysis and Its Applications. I. Fixed-point Theorems, Springer, New York, NY, USA, 1986.

[17] K. Deimling, Nonlinear Functional Analysis, Springer, Berlin, Germany, 1985. 